\title{
ORLICZ GEOMINIMAL SURFACE AREAS
}

\author{
SHUFENG YUAN, HAILIN JIN AND GANGSONG LENG
}

Abstract. In 1996, E. Lutwak extended the important concept of geominimal surface area to $L_{p}$ version, which serves as a bridge connecting a number of areas of geometry: affine differential geometry, relative differential geometry, and Minkowskian geometry. In this paper, by using the concept of Orlicz mixed volume, we extend geominimal surface area to the Orlicz version and give some properties and an isoperimetric inequalities for the Orlicz geominimal surface areas.

Mathematics subject classification (2010): 52A39, 52A40.

Keywords and phrases: Convex body, Orlicz mixed volume, Orlicz geominimal surface area, inequality.

\section{REFERENCES}

[1] F. Chen, J. Zhou And C. YAng, On the reverse Orlicz Busemann-Petty centroid inequality, Adv. in Appl. Math., 47, 4 (2011), 820-828.

[2] R. J. GARDner, Geometric Tomography, Second edition, Cambridge University Press, New York, 2006.

[3] R. J. Gardner, D. Hug And W. Weil, The Orlicz-Brunn-Minkowski Theory: A general framework, additions, and inequalities, J. Differential Geom., in press.

[4] C. Haberl, E. Lutwak, D. Yang And G. Zhang, The even Orlicz Minkowski problem, Adv. Math., 224 (2000), 2485-2510.

[5] Q. Huang And B. He, On the Orlicz. Minkowski problem for polytopes, Discrete Comput. Geom., 48 (2012), 281-297.

[6] A. Li And G. Leng, A new proof of the Orlicz Busemann-Petty centroid inequality, Proc. Amer. Math. Soc., 139, 4 (2011), 1473-1481.

[7] E. LutwaK, The Brunn-Minkowski-Firey theory I: Mixed volumes and the Minkowski problem, J. Differental Geom., 38 (1993), 131-150.

[8] E. Lutwak, The Brunn-Minkowski-Firey Theory II: Affine and Geominimal Surface Areas, Adv. Math., 118, 2 (1996), 244-294.

[9] E. Lutwak, D. Yang And G. Zhang, $L_{p}$ John ellipsoids, Proc. London Math. Soc., 90, 2 (2005), 497-520.

[10] E. LutwaK, D. Yang And G. Zhang, Orlicz projection bodies, Adv. Math., 223 (2010), 220-242.

[11] E. Lutwak, D. Yang And G. Zhang, Orlicz centroid bodies, J. Differential Geom., 84 (2010), $365-387$.

[12] M. Meyer And A. PAjor, On Santaló's inequality, in "Gemetric Aspects of Functional Analysis" (J. Lindenstrauss and V. D. Milman, Eds.), Springer Lecture Notes in Math, 1376, pp. 261-263, SpringerVerlag, New York/Berlin, 1989.

[13] M. Meyer And A. Pajor, On the Blaschke-Santaló inequality, Arch. Math., 55 (1990), 82-93.

[14] C. M. Petty, Geominimal surface area, Geom. Dedicata, 3 (1974), 77-97.

[15] R. SchneIder, Convex Bodies: The Brunn-Minkowski Theory, Cambridge University Press, Cambridge, 1993.

[16] G. ZHU, The Orlicz centroid inequality for star bodies, Adv. in Appl. Math., 48, 2 (2012), 432-445. 